\title{
Diversity of Nuclear Polyhedrosis Viruses on Lepidopteran Pests on Cruciferae in West Sumatera, Indonesia
}

\author{
Hidrayani and Martinius \\ \# Department of Plant Protection, Faculty of Agriculture, Andalas University, Indonesia \\ E-mail: hidrayani@unand.ac.id, martinius@unand.ac.id
}

\begin{abstract}
Nuclear Poyhedrosis Virus (NPV) is one of biological control agents that can be incorporated in Integrated Pest Management to support sustainable agriculture. A study was conducted to determine the diversity of NPV on lepidopteran pests on cruciferae in West Sumatera. Larvae of Spodoptera litura, Spodoptera exigua, Plutella xylostella, dan Crocidolomia binotalis were collected from cabbages, cauliflowers, broccoli, shallot, and leaf onion in three cruciferae production districts and then they were brought to laboratory for the observation of NPV infection. The results showed that there were only three larvae of Spodoptera exigua got infected by NPV called SeNPV. No other larvae of lepidopteran species collected were infected. Propagation of the SeNPV obtained was done but the pathogenisity was low with the mortality $12 \%$. It could be due to the low concentration of its polyhedra.
\end{abstract}

Keywords - NPV, lepidopteran pests, crucifera.

\section{INTRODUCTION}

In the high land of West Sumaterat, vegetables are very intensively cultured with intensive use of synthetic pesticides. It is very difficult for farmers not to rely on the pesticides even thought they have already known their negative impacts, such as : natural enemies are killed [4], [12] pests become resistant to pesticides [14] pests resurgence, secondary pest outbreak, problem of pesticides residue, and environmental pollution (Rauf et al 2000). In a program of West Sumatera government in developing organic farming, vegetables (cabbage, onion, broccoli, and others) is one of main comodity to be developed. However, in culturing the crops the main constrain is pest attaks mainly lepidopteran pests that could cause $100 \%$ loss [16], [7],[8]. A survey indicated that $89,2 \%$ farmers in West Sumatera mentioned that the main constraint in practising organic farming was plant pest and disease [5] while in organic farming the use of synthetic pesticides is not allowed.

To decrease the negative impact of synthetic pesticides and to avoid farmers' reliance on synthetic pesticides, Indonesian government has instructed to apply Integrated Pest Management (IPM) methode [17]. To support IPM, obtain agricultural product free from synthetic pesticides, and to conserve biodiversity, the use of synthetic pesticides should be decrease or not used at all. To achieve this points several alternative controls are needed.
One alternative control is biological control using natural enemies, i.e. parasitoid, predator and entomopathogens. In West Sumatera there have been lots of studies conducted on pests natural enemies, but none of scientist has done research on entomopathogen Nuclear Polyhedrosis Virus (NPV), while this bioological agent is very potential to develop. NPV has specific host, efective against pests already resistant to synthetic insecticides, safe for environment, and could be incorporated in IPM [18]. Thus, NPV could support IPM.

NPV is virus that kills insects through digestive system by attacking the suceptible cell nuclei. Some type of NPV have been studied. NPV infecting Spodoptera litura known as S1NPV has been studied by [1], infecting Spodoptera exigua known as Se-NPV, and infecting Helicoverpa armigera known as Ha- NPV have been studied [10]. Efectiveness of NPV bioinsecticide specific to army worm was field tested [2] and showed the result that the bioinsecticide could decrease $88 \%$ larval population, while those treated with monokrotofos insecticides with suggested dose could not decrease larval population but even increased $27 \%$ larval population.

Scientific study on NPV has never been studied in West Sumatera, while the infection of NPV on Lepidopteran pest was ever reported in a field especially on lepidopteran attacking cruciferae and onion. There are same Lepidopteran pest species attacking different species of crucifer could cause $100 \%$ loss. The same species of pest could also attack leaf onion or shallot causing total loss. With variety of host 
plants there could be variety of isolates of NPV with different effectiveness in controlling insect pests on their host plants. For that reason, a research on diversity of Nuclear Polyhedrosis Virus (NPV) as biological agent for Lepidopteran pests on vegetables has been conducted in West Sumatera. The objective of the research were to study the diversity of NPV infecting Lepidopteran pests attacking crucifers and leaf onion and shallot and to screen the best isolate to control Lepidopteran pests on that host plants.

\section{RESEARCH METHODOLOGY}

The research was conducted in field and laboratory from March-November 2013. Multiplication of hostplants. Host plants, broccoli and leaf onion were planted in pots and field as a feed for lepidopteran pests, Spodoptera litura, S. exigua, Crocidolomia pavonana, and Plutella xylostella

Exploration of Nuclear Polihedrosis Virus (NPV). Exploration of NPV was conducte in three central production of crucifers and leaf onion and shallots, Agam, Tanah Datar, and Solok district. In each district 2 subdistricts was determined. In each subdistrict some planting areas were taken. Lepidopteran larva were collected from crucifers: cabbage, cauliflower, and broccoli, and from leaf onion and shallot. Each species of larva was placed in plastic containers $(28 \mathrm{X} 20 \mathrm{~cm})$ and were labeled and brought to laboratory, Observation on larva infected was conducted every day. Dead larva infected by NPV is marked by larva hung upside down (hung with prologs) on top of container.

NPV propagation. Each of NPV isolate, based on species and location of host plants, was ground and added sterile water then smeared to the leaves surface of broccoli and leaf onion. The leaves were then fed to healthy larva. After larva were infected and dead, they were harvested and kept in freezer until they were used.

NPV Purification. Infected larva were ground and added with Sodium Dedocyl Sulphate $(0.1 \%)$ then centrifuged $2000 \mathrm{rpm}$ for 3 minutes. The debris like the crude tissue was discarded. The result was supernatant. Supernatant was then centrifuged $3000 \mathrm{rpm}$ for 30 minutes. The debris was discarded and the same work were repeated for ten 4 times. The end result was stock suspension of NPV polihedra

\section{RESULT AND DISCUSSION}

\section{A. Explorasion of Nuclear Polyhedrosis Virus (NPV)}

Exploration of NPV on Lepidopteran pests on vegetables in West Sumatera showed that there was only one isolate of NPV found i.e. the NPV infecting Spodoptera exigua known as SeNPV collected in Nagari Aie Angek, Tanah Datar District.

Table 1 shows that NPV was very rare found infecting lepidopteran larva in field. Out of 24 times larva collections, there were only 3 Spodoptera exigua larva found to be infected by NPV known as SeNPV. They were found on 21st visit on larva attacking leaf onion in Aie Angek Tanah Datar. During all visits we did not find larva with infected symptom.
TABLE I

EXSPLORATION OF NULEAR POLYHEDROSIS VIRUS (NPV) ON LEPIDOPTERAN PESTS ON VEGETABLES IN WEST SUMATERA

\begin{tabular}{|c|c|c|c|c|c|c|}
\hline No & $\stackrel{\mathscr{E}}{\tilde{\omega}}$ & 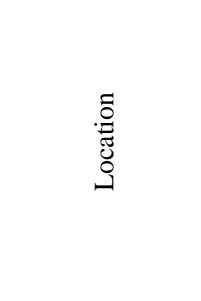 & 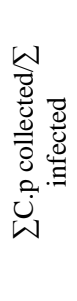 & 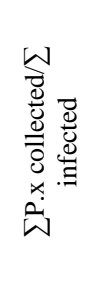 & 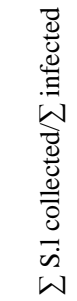 & 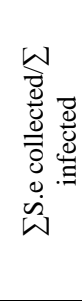 \\
\hline 1 & $\begin{array}{l}10-11 \\
\text { April } \\
2013\end{array}$ & $\begin{array}{l}\text { Solok: } \\
\text { Sungai Nanam } \\
\text { Simpang } \\
\text { Tanjung nan IV }\end{array}$ & $60 / 0$ & $20 / 0$ & - & $700 / 0$ \\
\hline 2 & $\begin{array}{l}24-25 \\
\text { April } \\
2013\end{array}$ & $\begin{array}{l}\text { Agam : } \\
\text { Batu Palano }\end{array}$ & - & $250 / 0$ & $200 / 0$ & $300 / 0$ \\
\hline 3 & $\begin{array}{l}1-2 \\
\text { Mei } 13\end{array}$ & $\begin{array}{l}\text { T Datar: } \\
\text { Aie Angek }\end{array}$ & $300 / 0$ & $200 / 0$ & $600 / 0$ & $400 / 0$ \\
\hline 4 & $\begin{array}{l}15-16 \\
\text { Mei } 13\end{array}$ & $\begin{array}{l}\text { Solok : } \\
\text { Sungai Nanam }\end{array}$ & - & $17 / 0$ & $30 / 0$ & $800 / 0$ \\
\hline 5 & $\begin{array}{l}29-30 \\
\text { Mei } 13\end{array}$ & $\begin{array}{l}\text { Agam : } \\
\text { Padang Lua }\end{array}$ & $300 / 0$ & $100 / 0$ & $700 / 0$ & $700 / 0$ \\
\hline 6 & $\begin{array}{l}4-5 \\
\text { Juni } 13\end{array}$ & $\begin{array}{l}\text { T. Datar } \\
\text { Aie Angek }\end{array}$ & $300 / 0$ & $150 / 0$ & $400 / 0$ & $500 / 0$ \\
\hline 7 & $\begin{array}{l}11-12 \\
\text { Juni } 13\end{array}$ & $\begin{array}{l}\text { Solok } \\
\text { Sungai Nanam }\end{array}$ & $5 / 0$ & $10 / 0$ & $200 / 0$ & $900 / 0$ \\
\hline 8 & $\begin{array}{l}\text { 19-20 } \\
\text { Juni } 13\end{array}$ & $\begin{array}{l}\text { Agam } \\
\text { Batu Palano }\end{array}$ & $300 / 0$ & $200 / 0$ & $400 / 0$ & $500 / 0$ \\
\hline 9 & $\begin{array}{l}26-27 \\
\text { Juni } 13\end{array}$ & $\begin{array}{l}\text { T. Datar } \\
\text { - Pandai Sikek }\end{array}$ & $300 / 0$ & $200 / 0$ & $400 / 0$ & $500 / 1$ \\
\hline 10 & $\begin{array}{l}\text { 17-18 } \\
\text { Juli } 13\end{array}$ & $\begin{array}{l}\text { Agam } \\
\text { - } \quad \text { Sariak } \\
\text { - } \text { Batu Palano } \\
\text { - } \text { Padang Lua }\end{array}$ & $196 / 0$ & $81 / 0$ & $129 / 0$ & $200 / 0$ \\
\hline 11 & $\begin{array}{l}31 \text { Juli- } \\
1 \text { Agus } \\
13\end{array}$ & $\begin{array}{l}\text { Solok } \\
\text { Sungai Nanam } \\
\text { Alahan Panjang }\end{array}$ & $182 / 0$ & - & $60 / 0$ & $301 / 0$ \\
\hline 12 & $\begin{array}{l}7-8 \\
\text { Agus } \\
13 \\
\end{array}$ & $\begin{array}{l}\text { T Datar } \\
\text { Pandai Sikek } \\
\text { Koto Laweh }\end{array}$ & $118 / 0$ & $111 / 0$ & $96 / 0$ & - \\
\hline 13 & $\begin{array}{l}12-13 \\
\text { Agus } \\
13\end{array}$ & $\begin{array}{l}\text { Agam } \\
\text { Padang Lua } \\
\text { Batu palano }\end{array}$ & - & - & $117 / 0$ & $172 / 0$ \\
\hline 14 & $\begin{array}{l}19-20 \\
\text { Agus } \\
13\end{array}$ & $\begin{array}{l}\text { T datar } \\
\text { Singgalang } \\
\text { Koto Laweh } \\
\text { Pandai Sikek }\end{array}$ & $201 / 0$ & - & $171 / 0$ & $219 / 0$ \\
\hline 15 & $\begin{array}{l}28-29 \\
\text { Agus } \\
13\end{array}$ & $\begin{array}{l}\text { Solok } \\
\text { Alahan Panjang } \\
\text { Simpang Tanj }\end{array}$ & - & - & $206 / 0$ & $302 / 0$ \\
\hline 16 & $\begin{array}{l}4-5 \\
\text { Sept } 13\end{array}$ & $\begin{array}{l}\text { Agam } \\
\text { Batu Palano } \\
\text { Padang Lua } \\
\text { Sariak }\end{array}$ & $256 / 0$ & $32 / 0$ & $197 / 0$ & $294 / 0$ \\
\hline 17 & $\begin{array}{l}9-10 \\
\text { Sept } 13\end{array}$ & $\begin{array}{l}\text { Solok } \\
\text { Sungai Nanam } \\
\text { Aie Dingin } \\
\end{array}$ & $102 / 0$ & - & $211 / 0$ & $201 / 0$ \\
\hline 18 & $\begin{array}{l}16-17 \\
\text { Sept } 13\end{array}$ & $\begin{array}{l}\text { T Datar } \\
\text { Aie Angek } \\
\text { Koto Laweh } \\
\text { Singgalang } \\
\end{array}$ & $123 / 0$ & - & $111 / 0$ & $203 / 0$ \\
\hline 19 & $\begin{array}{l}25-26 \\
\text { Sept } \\
13\end{array}$ & $\begin{array}{l}\text { Agam } \\
\text { Ampek Koto } \\
\text { Padang lua }\end{array}$ & $99 / 0$ & - & $99 / 0$ & $119 / 0$ \\
\hline 20 & $\begin{array}{l}2-3 \\
\text { Okt } 13\end{array}$ & $\begin{array}{l}\text { Solok } \\
\text { S Tanjung IV } \\
\text { Sungai Nanam } \\
\end{array}$ & $89 / 0$ & - & $101 / 0$ & $402 / 0$ \\
\hline 21 & $\begin{array}{l}7-8 \\
\text { Okt } 13\end{array}$ & $\begin{array}{l}\text { T Datar } \\
\text { Aie Angek } \\
\text { Pandai Sikek }\end{array}$ & $87 / 0$ & - & $72 / 0$ & $112 / 3$ \\
\hline
\end{tabular}




\begin{tabular}{|c|l|l|l|l|l|l|}
\hline 22 & $\begin{array}{l}14-15 \\
\text { Okt 13 }\end{array}$ & $\begin{array}{l}\text { Agam } \\
\text { Padang Lua } \\
\text { Batu Palano }\end{array}$ & $49 / 0$ & - & $91 / 0$ & $251 / 0$ \\
\hline 23 & $\begin{array}{l}28-29 \\
\text { Okt 13 }\end{array}$ & $\begin{array}{l}\text { T.Datar } \\
\text { Batu P AA } \\
\text { Singgalang }\end{array}$ & $187 / 0$ & & $79 / 0$ & $157 / 0$ \\
\hline 24 & $\begin{array}{l}3-4 \\
\text { Nov 13 }\end{array}$ & $\begin{array}{l}\text { Solok } \\
\text { Danau kembar } \\
\text { Sungai Nanam }\end{array}$ & $92 / 0$ & & $90 / 0$ & $89 / 0$ \\
\hline
\end{tabular}

Remark : C.p : Crocidolomia pavonanna, P.x : Plutella xylostella, S.1 : Spodoptera litura, S.e : Spodoptera exigua

Three larva infected were found after being reared in laboratory. SeNPV found in three S. exigua was then propagated by infecting healthy larva with 5 replications and each replication consisted of 10 larva. The plant used was leaf onion. The results of infection was presented in Table 2.

TABLE III

INFECTION OF SENPV ON SPODOPTERA EXIGUA ISOLATE AIE ANGEK TANAH DATAR

\begin{tabular}{|c|c|c|c|}
\hline Replication & $\begin{array}{c}\text { Number } \\
\text { larva }\end{array}$ & Number dead larva & $\begin{array}{c}\text { Mortality } \\
(\%)\end{array}$ \\
\hline 1 & 10 & 1 & 10 \\
\hline 2 & 10 & 2 & 20 \\
\hline 3 & 10 & 1 & 10 \\
\hline 4 & 10 & 0 & 0 \\
\hline 5 & 10 & 2 & 20 \\
\hline & \multicolumn{3}{|c}{} \\
\hline
\end{tabular}

Table 2 shows that the average mortality of S. exigua after being treated by SeNPV was $12 \%$, which indicated that the mortality was low. This could be the result of low concentration of polyhedral of SeNPV in S. exigua.

From 5 replications (50 larva), 6 infected larva with SeNPV were obtained. All 6 larva infected by SeNPV were then purified. Polyhedra as the result of purification could be seen under microscope with magnification 40X10 look at Fig.1.

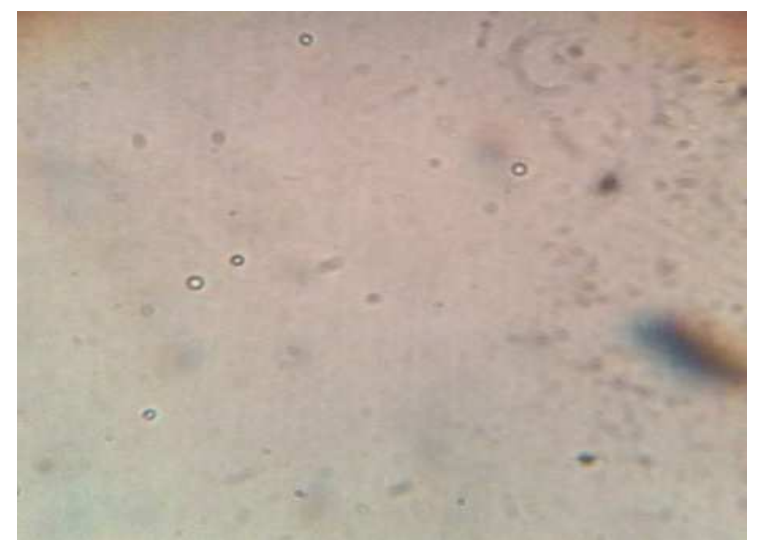

Fig. 1. Polyhedra of SeNPV isolate Aie Angek Kabupaten Tanah Datar

\section{IV.CONCLUSIONS}

Diversity of NPV in lepidopteran pests in West Sumatera was very low. The infection of NPV was only found in Spodoptera exigua attacking leaf onion, and none of infection occurred on other species of Lepidopteran. There were only 3 larva of Spodoptera exigua found to be infected by NPV known as SeNPV. Mortality of larva caused by SeNPV was low, i.e. $12 \%$. This indicated that consentrasi of polyhedra was low.

\section{REFERENCES}

[1] Arifin, M. 2002. Teknik produksi dan pemanfaatan bioinsektisida NPV untuk pengendalian ulat grayak pada kedelai. Dalam Suniardi et al. (Eds.). Prosiding Simposium Penelitian Tanaman Pangan IV: Tonggak Kemajuan Teknologi Produksi Tanaman Pangan, Komponen dan Paket Teknologi Produksi Palawija. Bogor. p 121134.

[2] Arifin,M,dan WIS Waskito. 1986. Kepekaan ulat grayak kedelai (Spodoptera litura) terhadap Nuclear Polyhedrosis Virus . Seminar hasil penelitian tanaman pangan. Puslitbangtan. Sukamandi, 16-18 Januari 1986.

[3] Hidrayani. 2011. Laporan Program Academik Recharging di Clemson University, Charleston. USA

[4] Hidrayani, Purnomo, Rauf A., Ridland PM, Hoffman AA. 2005. Pesticide applications on Java potato fields are ineffective in controlling leafminers, and have antagonistic effects on natural enemies of leafminers. Int. J. Pest Manage. 51 (3):181-187.

[5] Hidrayani dan Yunisman. 2006. Keragaman spesies dan bioekologi parasitoid telur trichogrammatidae di Sumatera Barat. Laporan penelitian Fundamental.

[6] Hidrayani. 2006. Pertanian organik: Pengelolaan hama dan penyakit salah satu kunci keberhasilan. Pidato ilmiah Dies natalis ke-52 Fakultas Pertanian Univ. Andalas. Padang. 30 November 2006.

[7] Hidrayani, Gani S., Salfitri A. 2006a. Kajian hama kubis bunga pada pertanaman yang diaplikasi dan tanpa insektisida. Laporan SP4.

[8] Hidrayani, Syam U, Fajri M. 2006b . Kajian parasitoid serangga hama tanaman kubis bunga (Brassica oleraceae L. var. Botrytis L.) pada pertanaman yang diaplikasi dan tanpa insektisida. Laporan SP4.

[9] Ignoffo CM, TL Couch. 1981. The Nuckeopolyhedrosis virus of Heliothis species as a microbial insecticide, p. 329-362. In H.D. Burges (Ed.). Microbial control of Pests and Plant diseases 19701980. Academic Press. London.

[10] Indrayani, IGAA dan AAA Gothama. 1991. Efisiensi pengendalian Helicoverpa armigera (Hubner) dengan NPV dan insektisida pada kapas. Pemberitaan Penelitian Tanaman Industri, 17 (2): 37-42

[11] JacksonDM, DE Lynn, JR Fuxa,BM Shepard, M Shapiro. 2009. Efficacy of entomophatogenic viruses on Pickleworm larvae and cell lines. J. Agric and Urban Entomol. Jaue.

[12] Oatman ER, Kennedy GG. 1976. Methomyl induced outbreak of Liriomyza sativae on tomato. J Econ Entomol 69(5):667-668.

[13] Okada,M. 1977. Studies on the utilization and mass production of Sl-NPV for control of the tobacco cutworm, Spodoptera litura F. Rev. Pl. Protec. Res. 10:102-128.

[14] Parella MP. 1982. A review of the history and taxonomy of ecomically important serpentine leafminers (Liriomyza spp) in California (Diptera: Agromyzidae). Pan Pac Entomol 58(4):302-308.

[15] Saphiro M, SE Salamouny, BM Separd, DM Jackson. 2010. Plant phenolics as Radiation protectants for the beet Armyworm (Lepidoptera: Noctuida) Nucleopolyhedrosisvirus. J.Agric.Urban Entomol. 26 (1)

[16] Sastrosiswojo S dan Sastrodihardjo S. 1986. Status of biological control of diamondback moth by introduction of parasitoid Diadegma eucerophaga in Indonesia. Di dalam: Talekar NS (ed). Diamondback Moth Management: Proceeding of Firts International Workshop. http://www.avrdc.org [download 20 Januari 2006]

[17] Sastrosiswojo, S. 1996. Biological control of diamondback moth in IPM system:Case study from Asia. BCPC symposium proceeding No.67: Biological control introduction.

[18] Starness,RL, CL Liu, PG Marrone. 1993. History, use, and future of microbial insecticides. American Entomologists. Summer: 83-91.

[19] Tanada Y., HK Kaya. 1993. Insect Pathology. Academic Press.Inc. New York. 665 p.

[20] Untung, K. 1993. Pengantar Pengelolaan Hama Terpadu. Gadjah Mada University Press. Hal 273.

[21] Utami. 1997. Virus Se-NPV atasi ulat grayak pada bawang merah. Trubus No. 337. Des 1997. Hal 74-76 